\title{
The Ranschburg effect: Modification of guessing strategies by context
}

\author{
JAMES V. HINRICHS and STEVEN P. MEWALDT \\ University of Iowa, Iowa City, Iowa 52242
}

\begin{abstract}
A guessing strategy interpretation of the Ranschburg effect-poor recall of repeated items in a short sequence-was tested by manipulating repetition frequency in the surrounding sequence. Three groups of 32 college students were tested with high-, low-, and mixed-repetition structure lists. The usual Ranschburg effect was obtained in the low-repetition group, but no significant differences were found on the high-repetition group. Performance in the mixed-repetition structure group was intermediate, but comparison of high and low sublists within the mixed condition revealed nearly identical performance for both sublists, more similar to the separate high-repetition group than to the low group.
\end{abstract}

The Ranschburg effect may be produced when subjects are required to recall immediately a short sequence containing at least one repeated item. The Ranschburg effect is defined as poorer recall of the repeated item, or of the experimental sequence containing the repeated item, compared to recall of a control sequence in which the item occurs only once. The items of particular interest within a given sequence (critical items) consist of both occurrences of the repeated item in experimental sequences and the unrepeated items occupying corresponding serial positions in control sequences. The earliest investigations of the Ranschburg effect have been summarized by McGeoch (1942), while more recent research and boundary conditions for the effect have been summarized in a scholarly review by Jahnke (1969).

The major difficulty posed by the Ranschburg effect is to understand why the repeated items, which should be remembered better than control elements according to conventional laws of learning and memory, are in fact recalled less well. A "guessing hypothesis" has been proposed (Hinrichs, Mewaldt, \& Redding, 1973) which contends that the Ranschburg effect is the result of a bias in the manner in which a subject guesses items he cannot remember. If an individual cannot store an entire sequence because its length exceeds his shortterm memory capacity, he may recall those items he was able to store and guess at the remainder. To maximize his performance, the subject will usually choose some type of guessing strategy, such as eliminating the items he is able to recall from the pool of possible items, in order to increase the probability of correctly guessing the remainder. If the presented sequence contained few or no repeats, the elimination strategy will enhance performance. However, if the item that the subject

We are greatly endebted to Cheryl Connelly and Frederick Liewehr for their assistance. Requests for reprints should be sent to James V. Hinrichs, Department of Psychology, University of Iowa, Iowa City, Iowa 52242. cannot recall is a second occurrence of a previously recalled item, and if he has eliminated items he has already recalled from the pool of possible responses, then the probability of correctly guessing the second occurrence will drop to zero, producing the Ranschburg effect. More generally, any tendency to avoid guessing previously recalled items will reduce recall performance on repeated items and improve performance on unrepeated items, resulting in an observed Ranschburg effect.

Hinrichs et al. (1973) attempted to manipulate the guessing strategy employed by the subject. They hypothesized that if subjects were exposed to many sequences containing repeated items other than the critical test items, they would be less likely to employ the restrictive guessing strategy. Reducing the use of this strategy should improve recall performance on experimental items by decreasing the bias against previously recalled items. The results showed a reduced Ranschburg effect consistent with predictions for experimental items in Serial Positions 3 and 6, but not for items in Positions 2 and 5. The results were interpreted as indicating that, when the critical items were presented later in the sequence, there was a greater probability that other noncritical repeated items had been presented and recalled. Thus, to the extent that the subjects' guessing strategy can be manipulated by contextual effects, later serial positions are more likely to show differences in the Ranschburg effect.

The primary question of interest in the present research is whether the subject adopts the restrictive guessing strategy in response to the context of a specific sequence, or whether he selects a strategy on the basis of some larger number of sequences. If an individual selects his guessing strategy in response to individual sequence context, then when exposed to a mixed list containing both high- and low-repetition structure sequences, he should exhibit differential performance on those sequences. Specifically, his performance on the high-repetition structure sequences of the mixed list should resemble his performance on a list of exclusively 
high-repetition structure sequences. Similarly, his performance on the low-repetition structure sequences of the mixed list should resemble his performance on a list of exclusively low-repetition structure sequences. If, however, the subject does not modify his strategy according to individual sequence context, but instead maintains a reasonably consistent strategy throughout a series of recall sequences, then no difference in the magnitude of the Ranschburg effect would be expected between his performance on high- and low-repetition structure sequences within the mixed list. In this case, the subject's performance on the mixed list should resemble his performance on either the high- or lowrepetition lists, or should resemble some intermediate level reflecting the extent to which the mixture of sequences contributed to his choice of strategy. The present study attempted to distinguish between these strategies.

\section{METHOD}

\section{Subjects}

The subjects were 96 introductory psychology students participating in order to fulfill a course requirement at the University of Iowa. The subjects were tested in small groups of 5-12 students and were assigned to one of eight treatment conditions in order of their appearance in the laboratory, according to a previously arranged schedule.

\section{Design and Materials}

Three repetition structure levels were utilized: low, mixed, and high, with 32 subjects per group. Within these three levels, subjects were required to recall sequences in two lists. The low-repetition structure condition consisted of two lists (Low A and Low B) in which the sequences surrounding the critical items contained no repeated items. The high-repetition structure condition consisted of two lists (High A and High B) which were composed of a combination of sequences containing one or two noncritical repeated items. The mixed condition consisted of four lists (Mixed A, B, C, and D) in which half the sequences in each list were taken from the low lists and half from the high lists.

There were 40 eight-item test sequences in each list, half of which were experimental sequences and half were control sequences. Half of each of these types of sequences contained critical items in Serial Positions 2 and 5, and half in Positions 3 and 6. In addition, in the mixed lists half of each of these four types of sequences was taken from the low lists, and half from the high lists. An additional 10 practice sequences of exactly the same construction as the control sequences, but which were not scored, preceded the 40 test sequences.

List Low A was constructed by sampling eight-digit sequences from a table of random permutations of the digits 0-9 (Moses \& Oakford, 1963) to form the control sequences. Experimental sequences were formed by changing the second critical item in 20 sequences randomly selected from the original list to a repeat of the first critical item. List Low B was constructed from List Low A by reversing the assignment of experimental and control sequences in the list. Consequently, each sequence occurred in both a control form, for half of the subjects, and in an experimental form, for the other half.

List High $\mathrm{A}$ was constructed by randomly selecting sequences of six digits from a table of random numbers, with the constraint that the six digits contain either one or two repetitions. A repetition was defined as the occurrence of an item in a previous serial position (i.e., 3933 and 2442 each contain two repeats). Eight-digit control sequences were constructed by inserting two different items in the appropriate positions (2-5 or $3-6)$; these critical digits could not occur elsewhere in the sequence. Again, experimental sequences were formed by changing the second critical digit of half the sequences to a repeat of the first, and List High B was formed by reversing the assignment of experimental and control sequences of List High $A$.

Mixed lists were constructed from the high and low lists. Specifically, Lists Mixed A and Mixed C each contained half of the experimental and control sequences from Lists Low A and High A, and Lists Mixed B and Mixed D each contained half the sequences from Lists Low B and High B. Lists were constructed so that experimental and control sequences maintained the same serial positions in the mixed versions as in the high and low versions.

In the construction of all lists, the following additional restrictions were observed: (1) Adjacent experimental sequences could not have the same repetition structure. (2) The same digit could not serve as the repeated critical digit in two consecutive experimental sequences. (3) Adjacent sequences could neither start nor end with the same digit. (4) No sequence could start with the same digit that had ended the preceding sequence.

\section{Apparatus and Procedure}

The stimulus sequences were presented visually by an Electronic Engineers Bina-View display controlled by a BRS Electronics digital logic system and paper tape reader. Stimuli were presented at the rate of $.65 \mathrm{sec} /$ item. Subjects were allowed $16 \mathrm{sec}$ for written recall, with a warning tone sounded after $14 \mathrm{sec}$ and $2 \mathrm{sec}$ before the presentation of the next sequence. A blank at the end of the sequence was used as the cue for recall. Subjects were instructed to recall the digits in the presented order by writing them left to right on an answer sheet containing eight boxes for each sequence. They were further instructed that they could guess or leave a blank in the position of an item about which they were uncertain. Complete sequences were covered with blank sheets of paper. Answer sheets for the first list were collected during the rest interval between the two lists.

\section{RESULTS}

Free recall scoring was employed for the critical item analysis, that is, a critical item was scored as correct if it appeared in any position in the response sequence. Following the procedure used by Jahnke (1969) and Hinrichs et al. (1973), items were scored as correct according to their input position, even if misplaced in recall. Single occurrences of an experimental item were assigned to the nearest serial position at which a repeated item should have occurred.

The results were then analyzed by a 2 by 2 by 2 by 2 by 3 factorial analysis of variance with position in the sequence (first or second), type of item (experimental or control), and list (first or second) as within-subject factors and list order (A-B or B-A) and repetition level (low, mixed, or high) as between-subject factors. Analysis of the recall performance was complicated by the observation of a four-way Item by List by Order by Repetition Level interaction $[F(2,90)=5.72, p<.01]$. Consequently, further analyses will concentrate on firstlist performance.

A position by item by list by repetition level analysis of first-list performance revealed the expected main effects of position $[F(1,90)=34.12, p<.001]$, with first-position recall better than second (.897 vs. .819$)$, and items $[F(1,90)=52.29, \mathrm{p}<.001]$, with control 
items recalled more often than repeated items $(.890$ vs. .826), demonstrating the Ranschburg effect. The main effects of list and repetition level were not significant $(p>.25)$ but two interactions were significant. First, as is usually observed, there was a significant Position by Items interaction $[\mathrm{F}(1,90)=19.57$, $\mathrm{p}<.001$ ], indicating a stronger Ranschburg effect in the second recall position (.872 vs. .766) than in the first (.908 vs. .886), but this difference did not vary with repetition level $(F<1)$. A separate analysis of variance using position of the critical items (2-5 vs. 3-6) yielded no significant Position by Items interaction $(F<1)$ or higher order interactions involving Position by Items ( $p>.25)$, further indicating no tendency for the Ranschburg effect to vary as a function of the joint position of the critical items. Second, and more important for the present investigation, there was a significant Items by Repetition Level interaction $[\mathrm{F}(2,90)=$ $5.07, \mathrm{p}<.01]$, described below. No other interactions were significant $(p>.05)$ except the Position by List interaction $(.025<p<.05)$, indicating only that the position effect noted above was stronger in List A than in List B.

The proportion of digits recalled correctly (out of 20 possible) for repeated and control critical items at each of the three repetition levels is presented in Table 1. Conservative Scheffe' follow-up tests of the Items by Repetition Level interaction indicated a significant Ranschburg effect for hoth the low-and mixedrepetition levels $(\mathrm{p}<.01$ and $\mathrm{p}<.05$, respectively), but no reliable difference for the high-repetition level ( $p>.10)$. The magnitude of the Ranschburg effect was significantly larger in the low-repetition group than in the high-repetition group $(\mathrm{p}<.05)$, but the magnitude of the effect in the mixed group did not reliably differ from either the high or the low group $(p>.05)$. Note that the decrease in the magnitude of the Ranschburg effect with increasing repetition level in the surrounding sequence is primarily attributable to an increase in recall of repeated items; recall of control items showed only a slight, nonsignificant performance drop from the low condition to the high condition.

Also shown in Table 1 is the most important comparison for the present investigation: the contrast of the relative magnitude of the Ranschburg effect for the high- and low-repetition sequences within the mixed

Table 1

Mean Proportion Correct Recall and Standard Errors of the Mean for Critical Items Across Three Repetition Levels

\begin{tabular}{llllll}
\hline & \multicolumn{2}{c}{ Repeated } & & \multicolumn{2}{c}{ Control } \\
\cline { 2 - 3 } \cline { 5 - 6 } Repetition Level & Mean & SE & & Mean & SE \\
\hline Low & .782 & .033 & .884 & .021 \\
Mixed & & & & \\
Low & .836 & .031 & .892 & .024 \\
High & .845 & .035 & .898 & .021 \\
Combined & .841 & .030 & .895 & .020 \\
High & .841 & .026 & .872 & .023 \\
\hline
\end{tabular}

condition. A separate analysis of variance on the mixed sequences showed a significant Ranschburg effect $[F(1,31)=17.92, p<.001]$ but no significant main effect of repetition level or the Items by Repetition Level interaction ( $F<1$ in both cases). The magnitude of the Ranschburg effect did not differ for high- and low-repetition sequences when those sequences were mixed in presentation although they did differ significantly when presented separately.

After converting performance scores to proportions, further comparisons can be made between the performance on the high- and low-repetition sequences in the homogeneous and mixed-repetition conditions (cf. Table 1). When only the low-repetition sequences in the mixed sequence condition were compared with the exclusively low-repetition condition, an Items by Repetition Level interaction was found $[F(1,60)=4.17$, $\mathrm{p}<.05$ ], with a larger Ranschburg effect in the lowrepetition condition (.884 vs. .782) than for the lowrepetition sequences in the mixed condition (.892 vs. .836). By contrast, the Items by Repetition Level interaction was not significant when comparing the high-repetition sequences in the mixed condition with the high-repetition condition $(\mathrm{F}<1)$.

\section{DISCUSSION}

The results replicate several previous investigations of influences on the Ranschburg effect and help define boundary conditions on the guessing interpretation of the Ranschburg effect. The present results replicate Experiments 2 and 3 of Hinrichs et al. (1973) by demonstrating a consistent context effect for repetition factors in short-term memory. That is, in accord with the guessing hypothesis, a large Ranschburg effect was produced when repeated items were embedded in a low-repetition surrounding sequence, but the magnitude of the effect was sharply reduced when the embedding sequence also contained repeated items. Also consistent with predictions from the guessing hypothesis, the Ranschburg effect was reduced primarily by increasing the recall of repeated critical items. In other words, increasing the likelihood of detecting and producing repeated items in recall apparently reduces the bias against guessing repeats when other items are forgotten. However, the within-sequence difference $(2-5$ vs. $3-6)$ in the magnitude of the Ranschburg effect obtained by Hinrichs et al. (1973) did not replicate, suggesting no greater influence of repetitions in the embedding sequence on reducing the Ranschburg effect in later positions than on earlier positions. In contrast to earlier speculation, no evidence supporting a shift in guessing bias during recall of a single sequence was obtained.

Interpretation of the obtained results begins with the observation that subjects appear to maintain a rather consistent response-selection strategy and change it slowly. The strategy of including or not including repeated responses does not seem to vary from sequence to sequence. Instead, the subject appears to adopt a strategy for dealing with forgotten items and maintains it until accumulating sufficient evidence to suggest a change. Initially, a strong bias against repeated responses is a dominant strategy. Indeed, it appears that at least some subjects maintain some reluctance to include repeated items in their recall even after encountering many sequences containing repeats, as indicated by consistently higher recall of control critical items in all reported studies even when several repeated elements are included in the sequences. On the other hand (and slightly contradictory), an increase in the proportion of sequences contain- 
ing repeated items is sufficient to reduce the magnitude of the Ranschburg effect in low-repetition sequences. Thus, the mixedrepetition group in the present experiment tended to perform more like the high-repetition group than the low. Although the threshold value is unknown (and probably varies considerably from individual to individual), the presentation of some sequences with more than an occasional repeated element is sufficient to reduce the Ranschburg effect, presumably by changing the guessing strategy employed by the subject. The results also further support the importance of the subject's ability to detect repetitions (Jahnke, 1969; Lee, 1976). When subjects do detect repetitions, they abandon (or at least markedly reduce) the use of a restricted guessing strategy.

\section{REFERENCES}

Hinrichs, J. V., Mewaldt, S. P., \& Redding, J. The
Ranschburg effect: Repetition and guessing factors in shortterm memory. Journal of Verbal Learning and Verbal Behavior, 1973, 12, 64-75.

Jahnke, J. C. The Ranschburg effect. Psychological Review, $1969,76,592-605$.

LEE, C. L. Short-term recall of repeated items and detection of repetitions in letter sequences. Journal of Experimental Psychology: Human Learning and Memory, 1976, 2, 120-127.

MCGEOCH, J. A. The psychology of human learning. New York: Longmans, Green, 1942.

Moses, L. E., \& OAKFORD, R. V. Tables of random permutations. Stanford: Stanford University Press, 1963.

(Received for publication September 3, 1976.) 